\title{
Nucleon polarizabilities in the perturbative chiral quark model
}

\author{
Yubing Dong ${ }^{* \dagger}$, Amand Faessler *, Thomas Gutsche *, Jan Kuckei *, \\ Valery E. Lyubovitskij *, Kem Pumsa-ard *, Pengnian Shen *† \\ * Institut für Theoretische Physik, Universität Tübingen, \\ Auf der Morgenstelle 14, D-72076 Tübingen, Germany \\ $\dagger$ Institute of High Energy Physics, Beijing 100049, P. R. China
}

(November 7, 2018)

\begin{abstract}
The nucleon polarizabilities $\alpha_{E}$ and $\beta_{M}$ are studied in the context of the perturbative chiral quark model. We demonstrate that meson cloud effects are sufficient to explain the electric polarizability of nucleon. Contributions of excite quark states to the paramagnetic polarizability are dominant and cancel the diamagnetic polarizability arising from the chiral field. The obtained results are compared to data and other theoretical predictions.
\end{abstract}

PACS: 12.39.Ki, 12.39.Fe, 13.60.Fz, 14.20.Dh

Keywords: Compton scattering, nucleon polarizabilities, chiral quark model, meson cloud 


\section{INTRODUCTION}

The nucleon polarizabilities $\alpha_{N}$ and $\beta_{N}$ with $N=p$ or $n$ are two of the fundamental observables which encode the electromagnetic structure of the nucleon. They can be determined from the differential cross sections of Compton scattering off the nucleon $\gamma+N \rightarrow \gamma+N$ (for a review see Refs. [1]- [5]). The idea to use Compton scattering to investigate the internal structure of the nucleon was formulated more than 50 years ago [6,7]. The first detailed measurement of the nucleon polarizabilities was performed in 1960 [8] by analyzing elastic $\gamma p$ scattering at $40-70 \mathrm{MeV}$. Compton scattering off the nucleon is constrained at very small energies by the Low-Gell-Mann-Goldberger low-energy theorem derived in Refs. $[9,10]$. It completely fixes the leading $O(1)$ and the next-to-leading $O(\omega)$ structure-independent contribution to the expansion of the scattering amplitude in powers of the photon frequency $\omega$. The leading term is known as the Thomson term and is expressed in terms of electron charge and nucleon mass only. The second term is proportional to the square of the anomalous magnetic moment of the nucleon. The nucleon polarizabilities characterize the quadratic terms in $\omega$ in the Compton scattering amplitude [11], of which the first structure-dependent terms come from Rayleigh scattering. Polarizabilities express the deformation of particles under the influence of quasi-static electric and magnetic fields. In particular, in the presence of an external electric $\vec{E}$ or magnetic $\vec{B}$ field the quark distribution of the nucleon becomes distorted, leading to the induced electric $\vec{d}=4 \pi \alpha_{E} \vec{E}$ or magnetic $\vec{\mu}=4 \pi \beta_{M} \vec{B}$ dipole moments, where the coefficients $\alpha_{E}$ and $\beta_{M}$ are the electric and magnetic polarizabilities of the nucleon. The interaction of the electric $\vec{d}$ and magnetic $\vec{\mu}$ dipole moments with the electromagnetic field leads to a change of the corresponding effective Hamiltonian. It describes the interaction of nucleons with electromagnetic fields generating quadratic terms in $\vec{E}$ and $\vec{B}$ :

$$
\Delta H_{\mathrm{eff}}=-\frac{4 \pi}{2}\left(\alpha_{E} \vec{E}^{2}+\beta_{M} \vec{B}^{2}\right) .
$$

The nucleon polarizabilities can be extracted from the Compton scattering cross section off the nucleon by comparison with

$$
\frac{d \sigma}{d \Omega} \sim\left[\left(\alpha_{E}+\beta_{M}\right)(1+\cos \theta)^{2}+\left(\alpha_{E}-\beta_{M}\right)(1-\cos \theta)^{2}\right]+\ldots
$$

where $\theta$ is the scattering angle. It was proposed already in the 50 s and later indicated in experimental and theoretical studies that the meson cloud effects give a significant contribution to the nucleon polarizabilities [5]. The sum $\alpha_{E}+\beta_{M}$ is constrained by the so-called Baldin sum rule [12] which establishes a connection to the integral over the unpolarized photo-absorption cross section. The current experimental data for nucleon polarizabilities are $[5]$ :

$$
\begin{aligned}
& \alpha_{E}^{p}=12.0 \pm 0.6 \times 10^{-4} \mathrm{fm}^{3}, \beta_{M}^{p}=1.9 \mp 0.6 \times 10^{-4} \mathrm{fm}^{3}, \\
& \alpha_{E}^{n}=12.5 \pm 1.7 \times 10^{-4} \mathrm{fm}^{3}, \beta_{M}^{n}=2.7 \mp 1.8 \times 10^{-4} \mathrm{fm}^{3} .
\end{aligned}
$$

Theoretical analyses of nucleon polarizabilities were done using different phenomenological approaches [13,14] (quark, soliton and Skyrme models) and methods of chiral perturbation theory [15-17]. 
A nonrelativistic approach in second-order perturbation theory gives the well-known sum rules for $\alpha_{E}=\alpha_{E}^{0}+\Delta \alpha_{E}$ and $\beta_{M}=\beta_{M}^{\text {para }}+\beta_{M}^{\text {dia }}$, where for example

$$
\alpha_{E}^{0}=2 \sum_{n \neq 0} \frac{|<n| d_{z}|0>|^{2}}{E_{n}-E_{0}}, \quad \Delta \alpha_{E}=Z_{N}^{2} \frac{e^{2}<r_{E}^{2}>^{N}}{3 m_{N}}
$$

are the leading contribution and the retardation correction to the electric polarizability. The magnetic polarizability contains the para- and diamagnetic contributions with

$$
\beta_{M}^{\text {para }}=2 \sum_{n \neq 0} \frac{|<n| \mu_{z}|0>|^{2}}{E_{n}-E_{0}}, \quad \beta_{M}^{\text {dia }}=-e^{2} \sum_{i=1}^{3} \frac{Q_{i}^{2}}{6 m_{i}}<0\left|\rho_{i}^{2}\right| 0>-\frac{<0\left|\vec{d}^{2}\right| 0>}{2 m_{N}} .
$$

Here $\mid n>$ and $E_{n}$ are eigenstate and energy of an excited state, $\vec{d}$ is the electric dipole moment, $d_{z}$ and $\mu_{z}$ are the $z$-components of the electric and magnetic moments, respectively, $Z_{N}$ and $m_{N}$ are the charge and the mass of nucleon, $Q_{i}, m_{i}$, and $\rho_{i}$ are the charge, mass and internal coordinate of the constituent quark, respectively. The problems associated with the use of the nonrelativistic sum rules have been discussed in [2,5,16-18]. In particular, the proton electric polarizability is predicted to be larger than the neutron one, which is in conflict with the central values of the experiment with $\alpha_{E}^{n} \geq \alpha_{E}^{p}$ [16]. It turns out that the inclusion of the $\Delta(1232)$-resonance is essential in the calculation of $\beta_{M}$. It gives a large positive contribution to the paramagnetic moment which compensates a relatively large negative diamagnetic polarizability $[16,18]$. Finally, the main problem is that the above nonrelativistic definition of the nucleon polarizabilities is not identical to the physical observables $\alpha_{E}$ and $\beta_{M}$ because of missing relativistic corrections [2,5]. Therefore, an accurate analysis of nucleon polarizabilities should be done within a truly relativistic approach.

Assuming $P$ -,$C$ - and $T$-invariance, the general amplitude for Compton scattering can be written in terms of six real structure dependent functions $A_{i}^{N}(\omega, \theta)$ with $i=1 \ldots 6$, where $\omega=\omega^{\prime}$ denotes the photon energy in the center-of-mass (CM) frame. The latter is specified by the following choice of momenta of initial $(p)$ and final $\left(p^{\prime}\right)$ nucleons and incoming $(k)$ and out-coming $\left(k^{\prime}\right)$ photon:

$$
p=(E,-\vec{k}), \quad p^{\prime}=\left(E,-\vec{k}^{\prime}\right), \quad k=(\omega, \vec{k}), \quad k^{\prime}=\left(\omega, \vec{k}^{\prime}\right) .
$$

For the polarization vectors of the real photon field it is convenient to choose the Coulomb gauge which is characterized by the conditions:

$$
\epsilon=(0, \vec{\epsilon}), \quad \epsilon^{* \prime}=\left(0, \vec{\epsilon}^{* \prime}\right), \quad \vec{k} \cdot \vec{\epsilon}=0, \quad \vec{k}^{\prime} \cdot \vec{\epsilon}^{* \prime}=0 .
$$

The $T^{N}$-matrix of Compton scattering is then written as [19]

$$
T^{N}=\sum_{i=1}^{6} A_{i}^{N}(\omega, \theta) S_{i}, \quad S_{1}=\vec{\epsilon}^{* \prime} \cdot \vec{\epsilon}, \quad S_{2}=\vec{\epsilon}^{* \prime} \cdot \hat{k} \vec{\epsilon} \cdot \hat{k}^{\prime}, \quad \ldots
$$

Here, $\epsilon, \hat{k}\left(\epsilon^{* \prime}, \hat{k}^{\prime}\right)$ are the polarization vector and the direction of the incoming (outgoing) photon with $\hat{k}=\vec{k} /|\vec{k}|$. The scattering angle $\theta$ is defined as $\cos \theta=\hat{k} \cdot \hat{k}^{\prime}$. In Eq. (7) only the first two terms are spin-independent and others are spin-dependent ones which contain 
the nucleon spin operator $\sigma$. The structure functions $A_{i}^{N}$ contain contributions from the pion-pole (anomalous) and from the remaining (regular) terms with

$$
A_{i}^{N}(\omega, \theta)=A_{i}^{N}(\omega, \theta)^{\pi^{0}-\text { pole }}+A_{i}^{N}(\omega, \theta)^{\mathrm{reg}}, \quad i=1 \ldots 6 .
$$

However, the $\pi^{0}$-pole does not contribute to the spin-independent structure functions $A_{1}^{N}$ and $A_{2}^{N}$. One can perform a low-energy expansion of the six nucleon structure functions $A_{i}^{N}(\omega, \theta)^{\text {reg }}$ in powers of the photon energy $\omega$. The explicit expressions of the expansion for the case of a proton target of mass $M_{N}$ with anomalous magnetic moment $\kappa^{p}$ can be found in Refs. [3,5]. The explicit expressions of the spin-independent functions $A_{1}^{N}(\omega, \theta)^{\mathrm{reg}}$ and $A_{2}^{N}(\omega, \theta)^{\text {reg }}$, which are expressed in terms of $\alpha_{E}$ and $\beta_{M}$, are [15]

$$
\begin{aligned}
A_{1}^{N}(\omega, \theta)^{\mathrm{reg}} & =-\frac{e^{2}}{m_{N}} Q_{N}^{2}+4 \pi\left(\alpha_{E}^{N}+\cos \theta \beta_{M}^{N}\right) \omega^{2} \\
& +\frac{4 \pi}{m_{N}}\left(\alpha_{E}^{N}+\beta_{M}^{N}\right)(1+\cos \theta) \omega^{3}+O\left(\omega^{4}, \frac{1}{m_{N}^{3}}\right), \\
A_{2}^{N}(\omega, \theta)^{\mathrm{reg}} & =\frac{e^{2}}{m_{N}^{2}} Q_{N}^{2} \omega-4 \pi \beta_{N}^{N} \omega^{2}-\frac{4 \pi}{m_{N}}\left(\alpha_{E}^{N}+\beta_{M}^{N}\right) \omega^{3}+O\left(\omega^{4}, \frac{1}{m_{N}^{3}}\right),
\end{aligned}
$$

where $Q_{N}$ is the nucleon charge.

In this work, a perturbative chiral quark model (PCQM) [20-22] is employed to study the spin-independent polarizabilities of nucleon, $\alpha_{N}$ and $\beta_{N}$. In the PCQM baryons are described by three relativistic valence quarks confined in a static potential, which are supplemented by a cloud of pseudoscalar Goldstone bosons, as required by chiral symmetry. This model has already been successfully applied to the charge and magnetic form factors of baryons, sigma terms, ground state masses of baryons, the electromagnetic $N \rightarrow \Delta$ transition, and other baryon properties [20-22]. A detailed discussion of the approach and a comparison to similar chiral quark models can be found in Ref. [20]. To calculate the nucleon polarizabilities we apply the heavy nucleon mass limit $m_{N} \rightarrow \infty$ where only the $\omega^{2}$ terms survive in the expansion of the regular amplitudes $A_{i=1,2}^{N}(\omega, \theta)^{\mathrm{reg}}$. From an evaluation of these terms we extract the nucleon polarizabilities.

The paper is organized as follows. In Sec. II we present an introduction to the PCQM. In Sec. III we calculate the nucleon polarizabilities in the PCQM. We discuss the numerical results and compare them to the predictions of other theoretical approaches. In Sec. IV we give our conclusions.

\section{THE PERTURBATIVE CHIRAL QUARK MODEL}

The perturbative chiral quark model is based on an effective chiral Lagrangian describing baryons by a core of the three valence quarks, moving in a central Dirac field with $V_{\text {eff }}(r)=$ $S(r)+\gamma^{0} V(r)$, where $r=|\vec{x}|$. In order to respect chiral symmetry, a cloud of Goldstone bosons $(\pi, K$ and $\eta)$ is included as small fluctuations around the three-quark core. Here we restrict to the $\mathrm{SU}(2)$ version of our approach and take into account only the pion cloud fluctuations. The total model Lagrangian is

$$
\mathcal{L}_{\text {eff }}=\mathcal{L}_{\text {inv }}+\mathcal{L}_{\chi S B}
$$


where $\mathcal{L}_{\text {inv }}$ is the chiral-invariant Lagrangian and $\mathcal{L}_{\chi S B}$ is the chiral symmetry breaking part. Up to second order in the pion field $\hat{\pi}=\pi_{i} \tau_{i}$ fluctuations, $\mathcal{L}_{\text {inv }}$ and $\mathcal{L}_{\chi S B}$ are

$$
\begin{gathered}
\mathcal{L}_{\text {inv }}(x)=\bar{\psi}(x)\left[i \not \partial-S(r)-\gamma^{0} V(r)\right] \psi(x)+\frac{1}{2}\left[\partial_{\mu} \hat{\pi}(x)\right]^{2}-\bar{\psi}(x) S(r) i \gamma^{5} \frac{\hat{\pi}(x)}{F} \psi(x) \\
+\bar{\psi}(x) \frac{\hat{\pi}^{2}(x)}{2 F^{2}} S(r) \psi(x), \\
\mathcal{L}_{\chi S B}(x)=-\bar{\psi}(x) \mathcal{M} \psi(x)-\frac{B}{2} \operatorname{Tr}\left[\hat{\pi}^{2}(x) \mathcal{M}\right] .
\end{gathered}
$$

Eq. (12) contains the mass contributions both for the quarks and pions, which explicitly break chiral symmetry. Lagrangian (11) contains the basic parameters: $F=88 \mathrm{MeV}$ is the pion decay constant in the chiral limit, $\mathcal{M}=\operatorname{diag}\{\hat{m}, \hat{m}\}$ is the mass matrix of current quarks (we restrict to the isospin symmetry limit $m_{u}=m_{d}=\hat{m}$ ) and $B=-<0|\bar{u} u| 0>/ F^{2}=$ $-<0|\bar{d} d| 0>/ F^{2}$ is the quark condensate constant. We rely on the standard picture of chiral symmetry breaking [23] and for the masses of pseudoscalar mesons we use the leading term in their chiral expansion (i.e. linear in the current quark mass). By construction, our effective chiral Lagrangian is consistent with the known low-energy theorems (GellMann-Okubo and Gell-Mann-Oakes-Renner relations, partial conservation of axial current (PCAC), Feynman-Hellmann relation between pion-nucleon $\sigma$-term and derivative of the nucleon mass, etc.).

We expand the quark field $\psi$ in the basis of potential eigenstates as

$$
\psi(x)=\sum_{\alpha} b_{\alpha} u_{\alpha}(\vec{x}) \exp \left(-i \mathcal{E}_{\alpha} t\right)+\sum_{\beta} d_{\beta}^{\dagger} v_{\beta}(\vec{x}) \exp \left(i \mathcal{E}_{\beta} t\right)
$$

where the sets of quark $\left\{u_{\alpha}\right\}$ and antiquark $\left\{v_{\beta}\right\}$ wave functions in orbits $\alpha$ and $\beta$ are solutions of the Dirac equation with the static potential $V_{\text {eff }}(r)$. The expansion coefficients $b_{\alpha}$ and $d_{\beta}^{\dagger}$ are the corresponding single quark annihilation and antiquark creation operators. In our calculation of matrix elements, we project quark diagrams on the respective baryon states. The baryon states are conventionally set up by the product of the SU(6) spin-flavor and $\mathrm{SU}(3)_{\mathrm{c}}$ color wave functions, where the nonrelativistic single quark spin wave function is simply replaced by the relativistic solution $u_{\alpha}(\vec{x})$ of the Dirac equation

$$
\left[-i \gamma^{0} \vec{\gamma} \cdot \vec{\nabla}+\gamma^{0} S(r)+V(r)-\mathcal{E}_{\alpha}\right] u_{\alpha}(\vec{x})=0,
$$

where $\mathcal{E}_{\alpha}$ is the single-quark energy of state $\alpha$.

For the description of baryon properties, we use the effective potential $V_{\text {eff }}(r)$ with a quadratic radial dependence $[20,21]$ :

$$
S(r)=M_{1}+c_{1} r^{2}, \quad V(r)=M_{2}+c_{2} r^{2}
$$

with the particular choice

$$
M_{1}=\frac{1-3 \rho^{2}}{2 \rho R}, \quad M_{2}=\mathcal{E}_{0}-\frac{1+3 \rho^{2}}{2 \rho R}, \quad c_{1} \equiv c_{2}=\frac{\rho}{2 R^{3}} .
$$


Here, $\mathcal{E}_{0}$ is the single-quark ground-state energy; $R$ and $\rho$ are parameters related to the ground-state quark wave function $u_{0}$ :

$$
u_{0}(\vec{x} ; i)=N_{0} \exp \left[-\frac{\vec{x}^{2}}{2 R^{2}}\right]\left(\begin{array}{c}
1 \\
i \rho \vec{\sigma}(i) \cdot \vec{x} / R
\end{array}\right) \chi_{s}(i) \chi_{f}(i) \chi_{c}(i),
$$

where $N_{0}=\left[\pi^{3 / 2} R^{3}\left(1+3 \rho^{2} / 2\right)\right]^{-1 / 2}$ is a normalization constant; $\chi_{s}, \chi_{f}, \chi_{c}$ are the spin, flavor and color quark wave functions, respectively. The index " $i$ " stands for the $i$-th quark. The constant part of the scalar potential $M_{1}$ can be interpreted as the constituent mass of the quark, which is simply the displacement of the current quark mass due to the potential $S(r)$. The parameter $\rho$ is related to the axial charge $g_{A}$ of the nucleon calculated in zeroth-order (or 3q-core) approximation:

$$
g_{A}=\frac{5}{3}\left(1-\frac{2 \rho^{2}}{1+\frac{3}{2} \rho^{2}}\right)
$$

Therefore, $\rho$ can be replaced by $g_{A}$ using the matching condition (17). The parameter $R$ is related to the charge radius of the proton in the zeroth-order approximation as

$$
<r_{E}^{2}>_{\mathrm{LO}}^{P}=\int d^{3} x u_{0}^{\dagger}(\vec{x}) \vec{x}^{2} u_{0}(\vec{x})=\frac{3 R^{2}}{2} \frac{1+\frac{5}{2} \rho^{2}}{1+\frac{3}{2} \rho^{2}} .
$$

In our calculations we use the value $g_{A}=1.25$. Therefore, we have only one free parameter in our model, that is $R$ or $\left\langle r_{E}^{2}>_{\mathrm{LO}}^{p}\right.$. In the numerical studies, $R$ is varied in the region from $0.55 \mathrm{fm}$ to $0.65 \mathrm{fm}$, which corresponds to a change of $\left\langle r_{E}^{2}>_{\mathrm{LO}}^{P}\right.$ from 0.5 to $0.7 \mathrm{fm}^{2}$. Note that for the given form of the effective potential (14) the Dirac equation (13) can be solved analytically [for the ground state see Eq.(16), for excited states see Ref. [22]]. In the Appendix we give details of the solutions to the Dirac equation for any excited state.

The expectation value of an operator $\hat{A}$ is then set up as:

$$
<\hat{A}>={ }^{B}<\phi_{0}\left|\sum_{n=1}^{\infty} \frac{i^{n}}{n !} \int d^{4} x_{1} \ldots \int d^{4} x_{n} T\left[\mathcal{L}_{I}\left(x_{1}\right) \ldots \mathcal{L}_{I}\left(x_{n}\right) \hat{A}\right]\right| \phi_{0}>_{c}^{B},
$$

where the state vector $\mid \phi_{0}>$ corresponds to the unperturbed three-quark state (3q-core). Superscript " $B$ " in the equation indicates that the matrix elements have to be projected onto the respective baryon states, whereas subscript " $c$ " refers to contributions from connected graphs only. $\mathcal{L}_{I}(x)$ of Eq. (19) refers to the quark-meson interaction Lagrangian:

$$
\mathcal{L}_{I}(x)=-\bar{\psi}(x) i \gamma^{5} \frac{\hat{\pi}(x)}{F} S(r) \psi(x)+\bar{\psi}(x) \frac{\hat{\pi}^{2}(x)}{2 F^{2}} S(r) \psi(x) .
$$

For the evaluation of Eq. (19) we apply Wick's theorem with the appropriate propagators for the quarks and pions.

For the quark field we use a Feynman propagator for a fermion in a binding potential with

$$
\begin{aligned}
i G_{\psi}(x, y) & =<\phi_{0}|T\{\psi(x) \bar{\psi}(y)\}| \phi_{0}> \\
& =\theta\left(x_{0}-y_{0}\right) \sum_{\alpha} u_{\alpha}(\vec{x}) \bar{u}_{\alpha}(\vec{y}) e^{-i \mathcal{E}_{\alpha}\left(x_{0}-y_{0}\right)}-\theta\left(y_{0}-x_{0}\right) \sum_{\beta} v_{\beta}(\vec{x}) \bar{v}_{\beta}(\vec{y}) e^{i \mathcal{E}_{\beta}\left(x_{0}-y_{0}\right)}
\end{aligned}
$$


In Refs. [20,21] we restricted the expansion of the quark propagator to its ground state with:

$$
i G_{\psi}(x, y) \rightarrow i G_{0}(x, y) \doteq u_{0}(\vec{x}) \bar{u}_{0}(\vec{y}) e^{-i \mathcal{E}_{0}\left(x_{0}-y_{0}\right)} \theta\left(x_{0}-y_{0}\right) .
$$

In Ref. [22] we investigated in addition the impact of excited states in the loops on the electromagnetic properties of baryons, the $\mathrm{N}-\Delta$ transition, the nucleon axial vector form factors, and meson-baryon sigma-terms. Here, we also include the excited quark states in the quark propagator of Eq. (21) to analyze their contribution to the nucleon polarizabilities. In our numerical calculation we include the following set of excited quark states: the first $p$-states ( $1 p_{1 / 2}$ and $1 p_{3 / 2}$ in the non-relativistic notation) and the second excited states $\left(1 d_{3 / 2}, 1 d_{5 / 2}\right.$ and $\left.2 s_{1 / 2}\right)$, i.e. we restrict to the low-lying excited states with energies smaller than the typical scale of $\Lambda=1 \mathrm{GeV}$. The justification for such an approximation can be found in our previous papers [22].

For the pions we adopt the free Feynman propagator with

$$
i \Delta_{i j}(x-y)=<0\left|T\left\{\pi_{i}(x) \pi_{j}(y)\right\}\right| 0>=\delta_{i j} \int \frac{d^{4} k}{(2 \pi)^{4} i} e^{-i k(x-y)} \Delta_{\pi}(k),
$$

where $\Delta_{\pi}(k)=\left[M_{\pi}^{2}-k^{2}-i 0^{+}\right]^{-1}$ is the pion propagator in the momentum space. Introduction of the electromagnetic field $A_{\mu}$ to the effective Lagrangian is accomplished by minimal substitution:

$$
\partial_{\mu} \psi \rightarrow D_{\mu} \psi=\partial_{\mu} \psi+i e Q A_{\mu} \psi, \quad \partial_{\mu} \pi_{i} \rightarrow D_{\mu} \pi_{i}=\partial_{\mu} \pi_{i}+e \varepsilon_{3 i j} A_{\mu} \pi_{j}
$$

where $Q=\operatorname{diag}\{2 / 3,-1 / 3\}$ is the $S U(2)$ quark charge matrix and $\varepsilon_{i j k}$ are the totally antisymmetric structure constants of $S U(2)$ (Levi-Civita tensor).

For the photon field $A_{\mu}$ we also include the usual kinetic term

$$
\mathcal{L}_{p h}=-\frac{1}{4} F_{\mu \nu} F^{\mu \nu} \quad \text { with } \quad F_{\mu \nu}=\partial_{\mu} A_{\nu}-\partial_{\nu} A_{\mu}
$$

The electromagnetic interaction of the system is

$$
\mathcal{L}_{e m}=-e A_{\mu} \bar{\psi} \gamma^{\mu} Q \psi-e A_{\mu} \varepsilon_{3 i j} \pi_{i} \partial^{\mu} \pi_{j}+\frac{e^{2}}{2} A_{\mu} A^{\mu}\left(\vec{\pi}^{2}-\pi_{0}^{2}\right) .
$$

\section{NUCLEON POLARIZABILITIES IN THE PCQM}

In this Section we apply the PCQM to calculate the spin-independent polarizabilities $\alpha_{E}$ and $\beta_{M}$ contained in the real Compton scattering amplitudes $A_{1}^{N}(\omega, \theta)^{\text {reg }}$ and $A_{2}^{N}(\omega, \theta)^{\text {reg }}$. In the heavy nucleon mass limit with $m_{N} \rightarrow \infty$ the structures of $A_{1}^{N}(\omega, \theta)^{\text {reg }}$ and $A_{2}^{N}(\omega, \theta)^{\text {reg }}$ amplitudes are sufficiently simplified as:

$$
\begin{aligned}
& \left.A_{1}^{N}(\omega, \theta)^{\mathrm{reg}}\right|_{m_{N} \rightarrow \infty}=4 \pi\left(\alpha_{E}^{N}+\cos \theta \beta_{M}^{N}\right) \omega^{2}, \\
& \left.A_{2}^{N}(\omega, \theta)^{\mathrm{reg}}\right|_{m_{N} \rightarrow \infty}=-4 \pi \beta_{N}^{N} \omega^{2} .
\end{aligned}
$$


This limit it well-justified in the PCQM and corresponds to the static three-quark bag with projection onto a nucleon state with finite three-momentum. Let us stress again that we do not intend to calculate the full Compton scattering amplitude $\gamma+N \rightarrow \gamma+N$ consistent with the relativistic theory. We just restrict to the evaluation of the spin-independent polarizabilities $\alpha_{N}$ and $\beta_{N}$. In the calculation of $\alpha_{N}$ and $\beta_{N}$ we proceed as follows. First we calculate the quark (one- and two-body) operators describing Compton scattering off a quark: $\gamma+q \rightarrow \gamma+q$. Then we project the set of these operators onto the nucleon states and extract $\alpha_{N}$ and $\beta_{N}$ from matching the result to Eq. (27).

The Feynman diagrams contributing to the process $\gamma+q \rightarrow \gamma+q$ (Compton scattering on the quark level) are shown in Figs.1-8: tree-level diagrams (Fig.1), one-body mesoncloud diagrams (Figs.2, 3, 4, 5 and 6) and two-body meson-cloud diagrams (Figs.7 and 8). Note, that the diagrams in Figs.4, 5, and 7 do not contribute to the real part of the spin-independent polarizabilities of the nucleon $\alpha_{E}$ and $\beta_{M}$. They only contribute to the spin-dependent polarizabilities. The intermediate ground state quark propagators contribute only in the diagrams of Figs.2 and 8 (both to $\alpha_{E}$ and to $\beta_{M}$ ). In the case of the diagrams of Fig.6 the intermediate ground quarks state contribute to the magnetic polarizabilities only. Excited intermediate quark states contribute to the tree-level (Fig.1) and meson-cloud diagrams (Figs.2 and 6).

For illustration we present the expressions for some typical diagrams contributing to the $T^{N}$ matrix. The contribution of the tree-level diagrams (Figs.1a and 1b) is:

$$
\begin{aligned}
& T_{1}^{N}=\epsilon_{m}^{* \prime} I_{1}^{m n} \epsilon_{n}, \quad I_{1}^{m n}=I_{1 \mathrm{a}}^{m n}+I_{1 \mathrm{~b}}^{m n}, \\
& I_{1 \mathrm{a}}^{m n}=e^{2} \sum_{i=1}^{3} \sum_{\alpha} \frac{V_{i}^{m}\left(\vec{k}^{\prime} ; \alpha\right) V_{i}^{n \dagger}(\vec{k} ; \alpha)}{\Delta E_{\alpha}-\omega-i 0^{+}}, ; I_{1 \mathrm{~b}}^{m n}=e^{2} \sum_{i=1}^{3} \sum_{\alpha} \frac{V_{i}^{n}(-\vec{k} ; \alpha) V_{i}^{m \dagger}\left(-\vec{k}^{\prime} ; \alpha\right)}{\Delta E_{\alpha}+\omega-i 0^{+}},
\end{aligned}
$$

where $(m, n)=1,2,3$, and $\Delta E_{\alpha}$ is the energy difference between the intermediate quark state (labeled by $\alpha$ ) and the initial (or final) quark ground state. The vertex function of the photon-quark coupling in Eq. (28) is

$$
V_{i}^{m}(\vec{k} ; \alpha)=\int d^{3} x \bar{u}_{0}(x ; i) \gamma^{m} Q_{i} u_{\alpha}(x ; i) e^{-i \vec{k} \cdot \vec{x}}
$$

where $u_{0}(x ; i)$ and $u_{\alpha}(x ; i)$ are the wave functions of ground and excited quark states; $Q_{i}$ is the charge number of the $i$-th quark. The amplitude $I_{1}^{m n}$ is proportional to $\sum_{i=1}^{3} Q_{i}^{2}$, resulting in 1 for proton and $2 / 3$ for neutron. If only the ground state is considered, the three-component of the vertex function is

$$
\vec{V}_{i}(\vec{k} ; 0)=i N_{0}^{2} \rho R\left(\pi R^{2}\right)^{3 / 2} e^{-\frac{k^{2} R^{2}}{4}} Q_{i}[\vec{\sigma}(i) \times \vec{k}],
$$

of which the quantities $\mathrm{R}, \rho$, and $N_{0}$ are defined in Sec. II. Tree-level diagrams constrained to include only intermediate ground state quarks do not contribute to the nucleon polarizabilities. This is due to the fact that $\Delta E_{\alpha}=0$ and, therefore, the expansion of $T_{1}^{m n}$ in powers of $\omega$ does not contain $\omega^{2}$ terms. Inclusion of the intermediate excited quark states in the tree-level diagrams leads to a contribution to the nucleon polarizabilities due to $\Delta E_{\alpha} \neq 0$. Moreover, only $\beta_{M}$ is affected by the tree-level diagrams, because they have the following spin structure 
$\vec{\sigma}(i) \cdot\left(\vec{k}^{\prime} \times \vec{\epsilon}^{* \prime}\right) \vec{\sigma}(i) \cdot(\vec{k} \times \vec{\epsilon})=\omega^{2}\left(\cos \theta \vec{\epsilon} \cdot \vec{\epsilon}^{* \prime}-\vec{\epsilon}^{* \prime} \cdot \hat{k} \vec{\epsilon} \cdot \hat{k}^{\prime}\right)+$ spin - dependent part.

As already mentioned before, only the meson-cloud diagrams of Figs.2, 3, 6, and 8 contribute to the spin-independent nucleon polarizabilities $\alpha_{E}$ and $\beta_{M}$. In other chiral approaches, the one-body diagrams of Figs.2 and 3 give rise to the dominant terms to $\alpha_{E}$ and $\beta_{M}$. In addition, in our approach we take into account two-body quark forces. Therefore, $\alpha_{E}$ and $\beta_{M}$ receive a contribution from the diagrams of Fig.8 which are the twobody analogue of the one-body diagrams of Fig.2. The diagrams of Fig.6 evaluated for the nucleon polarizabilities are suppressed. For example, the explicit expression of the diagrams in Fig. 2 to the $T^{N}$ matrix is given by

$$
T_{2}^{N}=\epsilon_{m}^{* \prime} I_{2}^{m n} \epsilon_{n}, \quad I_{2}^{m n}=I_{2 \mathrm{a}}^{m n}+I_{2 \mathrm{~b}}^{m n}+I_{2 \mathrm{c}}^{m n},
$$

where

$$
\begin{aligned}
I_{2 a}^{m n}+I_{2 b}^{m n} & =-\frac{e^{2}}{F^{2}} \sum_{i=1}^{3} \sum_{\alpha} \int \frac{d^{4} q}{(2 \pi)^{4} i} \\
& \times\left[P_{\alpha}\left(\vec{q}-\vec{k}^{\prime} ; i\right) \Delta_{\pi}\left(q-k^{\prime}\right) \Delta_{\pi}(q) \Delta_{\pi}(q-k) P_{\alpha}^{\dagger}(\vec{q}-\vec{k} ; i) \frac{\left(2 q-k^{\prime}\right)^{m}(2 q-k)^{n}}{q_{0}+\Delta E_{\alpha}-\omega-i 0^{+}}\right. \\
& \left.+P_{\alpha}(\vec{q}+\vec{k} ; i) \Delta_{\pi}\left(q+k^{\prime}\right) \Delta_{\pi}(q) \Delta_{\pi}(q+k) P_{\alpha}^{\dagger}\left(\vec{q}+\vec{k}^{\prime} ; i\right) \frac{\left(2 q+k^{\prime}\right)^{m}(2 q+k)^{n}}{q_{0}+\Delta E_{\alpha}+\omega-i 0^{+}}\right] \\
I_{2 c}^{m n} & =-\delta^{m n} \frac{e^{2}}{F^{2}} \sum_{i=1}^{3} \sum_{\alpha} \int \frac{d^{4} q}{(2 \pi)^{4} i} \frac{1}{q_{0}+\frac{\Delta k_{0}}{2}+\Delta E_{\alpha}-i 0^{+}} \\
& \times P_{\alpha}\left(\vec{q}-\frac{\Delta \vec{k}}{2} ; i\right) \Delta_{\pi}\left(q-\frac{\Delta k}{2}\right) \Delta_{\pi}\left(q+\frac{\Delta k}{2}\right) P_{\alpha}^{\dagger}\left(\vec{q}+\frac{\Delta \vec{k}}{2} ; i\right)
\end{aligned}
$$

with $q$ being the pion momentum and $\Delta k=k^{\prime}-k$. Here, $P_{\alpha}(\vec{k} ; i)$ is the quark-pion vertex function

$$
P_{\alpha}(\vec{k} ; i)=\int d^{3} x \bar{u}_{0}(x ; i) \gamma_{5} S(r) u_{\alpha}(x ; i) e^{i \vec{k} \cdot \vec{x}}
$$

where $S(r)$ is the scalar part of the confinement potential. In particular, when $\alpha=0$, we have

$$
\begin{aligned}
P_{0}(\vec{k} ; i) & =-\frac{3}{10} g_{A} F_{\pi N N}\left(\vec{k}^{2}\right) \vec{\sigma}(i) \cdot \vec{k} \\
F_{\pi N N}\left(\vec{k}^{2}\right) & =\exp \left(-\frac{\vec{k}^{2} R^{2}}{4}\right)\left[1+\frac{\vec{k}^{2} R^{2}}{8}\left(1-\frac{5}{3 g_{A}}\right)\right],
\end{aligned}
$$

where $F_{\pi N N}\left(\vec{k}^{2}\right)$ is the $\pi N N$ form factor normalized to unity at zero recoil, $\vec{k}^{2}=0$.

Our results for the proton and neutron spin-independent polarizabilities $\alpha_{E}$ and $\beta_{M}$ are contained in Figs.9-18. In Figs.9-12 we show the sensitivity of the proton (Figs.9 and 11) and neutron polarizabilities (Figs.10 and 12) to a variation in the model parameter $<r_{E}^{2}>_{\text {LO }}^{p}$. In Figs.13-18 we demonstrate the contribution of the individual excited states in the tree-level (Figs.13 and 14) and in the meson-cloud (Figs.15-18) diagrams to $\beta_{M}^{p}$ and $\beta_{M}^{n}$. 
The contribution of the tree-level diagrams to $\beta_{M}$ grows linearly when the size parameter $\left\langle r_{E}^{2}\right\rangle_{\mathrm{LO}}^{p}$ increases. Results for the meson-cloud diagrams are not changed so much as function of $\left\langle r_{E}^{2}>_{\mathrm{LO}}^{p}\right.$ (when we restrict to the inclusion of the ground state quark propagators only) and grows (decreases) in $\alpha_{E}\left(\beta_{M}\right)$ when $\left\langle r_{E}^{2}>_{\mathrm{LO}}^{p}\right.$ increases.

Now we discuss the numerical results for the nucleon polarizabilities in the case of the central value $\left\langle r_{E}^{2}>_{\mathrm{LO}}^{p}=0.6 \mathrm{fm}^{2}\right.$ obtained in previous phenomenology [20-22]. In Table I we compare our results to the data and to other theories (ChPT [15], ChPT + dispersion relation (DR) constraints [17]). In Table II we present the individual contributions of the different diagrams to the nucleon polarizabilities, while also listing the separate results for the ground and excited quark states in the loops. It can be seen that the tree-level diagrams and the meson cloud diagrams of Fig. 6 do not contribute to the electric polarizabilities. The contribution of the tree-level diagrams to the magnetic polarizabilities is solely due to the inclusion of the excited quark states. As already indicated, the excited state contributions in the tree-level diagrams are for the case of the neutron $2 / 3$ of the result of the proton. The total predicted value for $\beta_{M}^{p}$ is larger than that of $\beta^{n}$. Finally, we found that only very small differences appear in the contribution of the six diagrams of Fig.6 to $\beta_{M}$, which changes slightly from 0.085 for the proton to 0.089 for the neutron. In our model calculations there is no remarkable difference between the proton and neutron cases for the pion-cloud diagrams. Thus, our results show that $\alpha_{E}^{p}=\alpha_{E}^{n}$. Moreover, the large difference between proton and neutron arises for $\beta_{M}$, where the tree-level diagrams of intermediate excited quark states differ strongly for both cases.

\section{CONCLUSIONS}

In this work we apply the perturbative chiral quark model to the description of the nucleon spin-independent polarizabilities $\alpha_{E}$ and $\beta_{M}$. It has been previously verified that the model is successful in the explanation of many aspects of nucleon properties [14-18], such as the magnetic moments, the axial vector form factor, the $N \rightarrow \Delta$ transition amplitude, the meson-nucleon sigma-term and $\pi N$ nucleon scattering. Here, we find that the model can reasonably reproduce the electric polarizabilities of proton and neutron without the use of any additional free parameters. The magnetic polarizabilities, in particular for the proton, are overestimated when compared to the present data. This effect is known from experience of other theoretical calculations.

In the perturbative chiral quark model it is found that without excited quark states in the loops already reasonable value for $\alpha_{E}$ and a negative one for $\beta_{M}$ are obtained. Then $\beta_{M}$ behaves like a diamagnetic polarizability. This finding also appears in the dispersion relation analysis, in other chiral quark model calculations and in leading-order relativistic chiral perturbative theory (see discussion in Ref. [5]). When we include the excite states in the quark propagators we get a paramagnetic polarizability $\beta_{M}$ arising from the tree-level diagrams. Our value for $\beta_{M}^{p}$ is overestimated in comparison with the data. This trend is also seen in heavy baryon chiral perturbative theory with the missing $\Delta(1232)$ contribution in terms of a "small scale expansion". From a detailed analysis we find that the intermediate excited quark states are important to generate a positive value for $\beta_{M}$. In fact, the sizeable contribution of excited states has already been elaborated in our calculation of the $\Delta \rightarrow N$ transition amplitude and in the case of the nucleon axial charge [22]. Comparing each 
contribution of the five intermediate excited states, we find that the effects of $1 p_{3 / 2}$ and $1 p_{1 / 2}$ are more pronounced than the effects of the other three resonances. For the neutron case, the main contribution, to the paramagnetic polarizability $\beta_{M}$ from the excited states of the Born terms, is proportional to $\sum_{j=1}^{3} Q_{j}^{2}$. Thus, the paramagnetic polarizability of the neutron is smaller than that of the proton target. For the observable $\alpha_{E}$, the one-pion loop contributions, both from the ground state and from the excited states, are not sensitive to isospin. Consequently, we have $\alpha_{E}^{p} \sim \alpha_{E}^{n}$ in the present calculation.

\section{ACKNOWLEDGMENTS}

This work was supported by the DFG under contracts FA67/25-3 and GRK683. This research is also part of the EU Integrated Infrastructure Initiative Hadron physics project under contract number RII3-CT-2004-506078 and the President grant of Russia "Scientific Schools" No. 1743.2003. The support from the National Natural Science Foundations of China is also appreciated. K.P. thanks the Development and Promotion of Science and Technology Talent Project (DPST), Thailand for financial support. Y.Dong and P.Shen thank the Institut für Theoretische Physik, Universität Tübingen, for their hospitality. 


\section{APPENDIX A. SOLUTIONS OF THE DIRAC EQUATION FOR THE EFFECTIVE POTENTIAL}

In this section we indicate the solutions to the Dirac equation with the effective potential $V_{\text {eff }}(r)=S(r)+\gamma^{0} V(r)$. The scalar $S(r)$ and time-like vector $V(r)$ parts are given by

$$
\begin{aligned}
& S(r)=M_{1}+c_{1} r^{2}, \\
& V(r)=M_{2}+c_{2} r^{2},
\end{aligned}
$$

with the particular choice

$$
M_{1}=\frac{1-3 \rho^{2}}{2 \rho R}, \quad M_{2}=\mathcal{E}_{0}-\frac{1+3 \rho^{2}}{2 \rho R}, \quad c_{1} \equiv c_{2}=\frac{\rho}{2 R^{3}} .
$$

The quark wave function $u_{\alpha}(\vec{r})$ in state $\alpha$ with eigen-energy $\mathcal{E}_{\alpha}$ satisfies the Dirac equation

$$
\left[-i \vec{\alpha} \vec{\nabla}+\beta S(r)+V(r)-\mathcal{E}_{\alpha}\right] u_{\alpha}(\vec{r})=0 .
$$

Solutions of the Dirac spinor $u_{\alpha}(\vec{r})$ can be written in the form [24]

$$
u_{\alpha}(\vec{r})=N_{\alpha}\left(\begin{array}{c}
g_{\alpha}(r) \\
i \vec{\sigma} \cdot \hat{r} f_{\alpha}(r)
\end{array}\right) \mathcal{Y}_{\alpha}(\hat{r}) \chi_{f} \chi_{c} .
$$

For the particular choice of the potential the radial functions $g$ and $f$ satisfy the form

$$
g_{\alpha}(r)=\left(\frac{r}{R_{\alpha}}\right)^{l} L_{n-1}^{l+1 / 2}\left(\frac{r^{2}}{R_{\alpha}^{2}}\right) e^{-\frac{r^{2}}{2 R_{\alpha}^{2}}},
$$

where for $j=l+\frac{1}{2}$

$$
f_{\alpha}(r)=\rho_{\alpha}\left(\frac{r}{R_{\alpha}}\right)^{l+1}\left[L_{n-1}^{l+3 / 2}\left(\frac{r^{2}}{R_{\alpha}^{2}}\right)+L_{n-2}^{l+3 / 2}\left(\frac{r^{2}}{R_{\alpha}^{2}}\right)\right] e^{-\frac{r^{2}}{2 R_{\alpha}^{2}}}
$$

and for $j=l-\frac{1}{2}$

$$
f_{\alpha}(r)=-\rho_{\alpha}\left(\frac{r}{R_{\alpha}}\right)^{l-1}\left[\left(n+l-\frac{1}{2}\right) L_{n-1}^{l-1 / 2}\left(\frac{r^{2}}{R_{\alpha}^{2}}\right)+n L_{n}^{l-1 / 2}\left(\frac{r^{2}}{R_{\alpha}^{2}}\right)\right] e^{-\frac{r^{2}}{2 R_{\alpha}^{2}}} .
$$

The label $\alpha=(n l j m)$ characterizes the state with principle quantum number $n=1,2,3, \ldots$, orbital angular momentum $l$, total angular momentum $j=l \pm \frac{1}{2}$ and projection $m$. Due to the quadratic nature of the potential the radial wave functions contain the associated Laguerre polynomials $L_{n}^{k}(x)$ with

$$
L_{n}^{k}(x)=\sum_{m=0}^{n}(-1)^{m} \frac{(n+k) !}{(n-m) !(k+m) ! m !} x^{m} .
$$

The angular dependence $\left(\mathcal{Y}_{\alpha}(\hat{r}) \equiv \mathcal{Y}_{l m j}(\hat{r})\right)$ is defined by

$$
\mathcal{Y}_{l m j}(\hat{r})=\sum_{m_{l}, m_{s}}\left(l_{l} \frac{1}{2} m_{s} \mid j m\right) Y_{l m_{l}}(\hat{r}) \chi_{\frac{1}{2} m_{s}}
$$


where $Y_{l m_{l}}(\hat{r})$ is the usual spherical harmonic. Flavor and color parts of the Dirac spinor are represented by $\chi_{f}$ and $\chi_{c}$, respectively.

The normalization constant is obtained from the condition

$$
\int_{0}^{\infty} d^{3} \vec{r} u_{\alpha}^{\dagger}(\vec{r}) u_{\alpha}(\vec{r})=1
$$

which results in

$$
N_{\alpha}=\left[2^{-2(n+l+1 / 2)} \pi^{1 / 2} R_{\alpha}^{3} \frac{(2 n+2 l) !}{(n+l) !(n-1) !}\left\{1+\rho_{\alpha}^{2}\left(2 n+l-\frac{1}{2}\right)\right\}\right]^{-1 / 2},
$$

The two coefficients $R_{\alpha}$ and $\rho_{\alpha}$ are of the form

$$
\begin{aligned}
R_{\alpha} & =R\left(1+\Delta \mathcal{E}_{\alpha} \rho R\right)^{-1 / 4}, \\
\rho_{\alpha} & =\rho\left(\frac{R_{\alpha}}{R}\right)^{3},
\end{aligned}
$$

and are related to the Gaussian parameters $\rho$ and $R$ of Eq. (16). The quantity $\Delta \mathcal{E}_{\alpha}=\mathcal{E}_{\alpha}-\mathcal{E}_{0}$ is the difference between the energy of state $\alpha$ and the ground state. $\Delta \mathcal{E}_{\alpha}$ depends on the quantum numbers $n$ and $l$ and is related to the parameters $\rho$ and $R$ by

$$
\left(\Delta \mathcal{E}_{\alpha}+\frac{3 \rho}{R}\right)^{2}\left(\Delta \mathcal{E}_{\alpha}+\frac{1}{\rho R}\right)=\frac{\rho}{R^{3}}(4 n+2 l-1)^{2} .
$$




\section{REFERENCES}

[1] V. A. Petrunkin, Fiz. Elem. Chast. Atom. Yadra 12 (1981) 692.

[2] A. I. Lvov, Int. J. Mod. Phys. A 8 (1993) 5267.

[3] D. Drechsel, B. Pasquini and M. Vanderhaeghen, Phys. Rept. 378 (2003) 99 [arXiv:hep$\mathrm{ph} / 0212124]$.

[4] F. Wissmann, Springer Tracts in Modern Physics 200 (2004) 1.

[5] M. Schumacher, arXiv:hep-ph/0501167.

[6] R. G. Sachs, L. L. Foldy, Phys. Rev. 80 (1950) 824,

[7] Yu. A. Aleksandrov, P. I. Bondarenko, Zh. Eksp. Theor. Fiz. 31 (1956) 726 [Sov. Phys. - JETPH 4 (1957) 612].

[8] V. I. Gol'danskii, O. A. Karpukhin, A. V. Kutsenko, V. V. Pavlovskaya, Zh. Eksp. Teor. Fiz 38 (1960) 1695 [Sov. Phys. - JEPT 11 (1960) 1223]; Nucl. Phys. 18 (1960) 473.

[9] F. E. Low, Phys. Rev. 96 (1954) 1428.

[10] M. Gell-Mann and M. L. Goldberger, Phys. Rev. 96 (1954) 1433.

[11] V. A. Petrun'kin, Zh. Eksp. Theor. Fiz. 40 (1961) 1148 [Sov. Phys. - JETPH 13 (1961) 808].

[12] A. M. Baldin, Nucl. Phys. 18 (1960) 310.

[13] V. A. Petrun'kin, Nucl. Phys. 55 (1964) 197; T. E. O. Ericson, J. Hüfner, Nucl. Phys. B 57 (1973) 604; Annals Phys. 95 (1975) 170.

[14] P. C. Hecking, G. F. Bertsch, Phys. Lett. B 99 (1981) 237; A. Schafer, R. Muller, D. Vasak and W. Greiner, Phys. Lett. B 143 (1984) 323; G. Dattoli, G. Matone and D. Prosperi, Lett. Nuovo Cim. 19, 601 (1977); D. Drechsel and A. Russo, Phys. Lett. B 137, 294 (1984); M. De Sanctis and D. Prosperi, Nuovo Cim. A 103, 1301 (1990); S. Capstick and B. D. Keister, Phys. Rev. D 46, 84 (1992) [Erratum-ibid. D 46, 4104 (1992)]; M. Traini and R. Leonardi, Phys. Lett. B 334, 7 (1994); H. Liebl and G. R. Goldstein, Phys. Lett. B 343, 363 (1995) [arXiv:hep-ph/9411230]; R. Weiner and W. Weise, Phys. Lett. B 159 (1985) 85; N. N. Scoccola and W. Weise, Nucl. Phys. A 517, 495 (1990); N. N. Scoccola and W. Weise, Phys. Lett. B 232, 287 (1989); W. Broniowski, M. K. Banerjee and T. D. Cohen, Phys. Lett. B 283, 22 (1992); E. M. Nyman, Phys. Lett. B 142, 388 (1984); M. Chemtob, Nucl. Phys. A 473, 613 (1987); S. Scherer and P. J. Mulders, Nucl. Phys. A 549, 521 (1992).

[15] V. Bernard, N. Kaiser and U. G. Meissner, Phys. Rev. Lett. 67 (1991) 1515; V. Bernard, N. Kaiser and U. G. Meissner, Nucl. Phys. B 373 1992) 346; V. Bernard, N. Kaiser, A. Schmidt and U. G. Meissner, Phys. Lett. B 319 (1993) 269 [arXiv:hep-ph/9309211]; D. Babusci, G. Giordano and G. Matone, Phys. Rev. C 55 (1997) 1645; T. R. Hemmert, B. R. Holstein, G. Knochlein and S. Scherer, Phys. Rev. D 55 (1997) 2630 [arXiv:nuclth/9608042]; T. R. Hemmert, B. R. Holstein, G. Knochlein and D. Drechsel, Phys. Rev. D 62 (2000) 014013 [arXiv:nucl-th/9910036].

[16] T. R. Hemmert, B. R. Holstein and J. Kambor, Phys. Rev. D 55 (1997) 5598 [arXiv:hepph/9612374]; B. R. Holstein, arXiv:hep-ph/9710548.

[17] A. I. Lvov, Phys. Lett. B 304 (1993) 29.

[18] N. C. Mukhopadhyay, A. M. Nathan and L. Zhang, Phys. Rev. D 47 (1993) 7.

[19] V. I. Ritus, Zh. Eksp. Theor. Fiz. 33 (1957) 1264 [Sov. Phys. - JETPH 6 (1957) 972]. 
[20] V. E. Lyubovitskij, T. Gutsche and A. Faessler, Phys. Rev. C 64, 065203 (2001) [arXiv:hep-ph/0105043].

[21] V. E. Lyubovitskij, T. Gutsche, A. Faessler and E. G. Drukarev, Phys. Rev. D 63, 054026 (2001) [arXiv:hep-ph/0009341]; V. E. Lyubovitskij, T. Gutsche, A. Faessler and R. Vinh Mau, Phys. Lett. B 520, 204 (2001) [arXiv:hep-ph/0108134]; Phys. Rev. C 65, 025202 (2002) [arXiv:hep-ph/0109213]; V. E. Lyubovitskij, P. Wang, T. Gutsche and A. Faessler, Phys. Rev. C 66, 055204 (2002) [arXiv:hep-ph/0207225]; F. Simkovic, V. E. Lyubovitskij, T. Gutsche, A. Faessler and S. Kovalenko, Phys. Lett. B 544, 121 (2002) [arXiv:hep-ph/0112277].

[22] S. Cheedket, V. E. Lyubovitskij, T. Gutsche, A. Faessler, K. Pumsa-ard and Y. Yan, Eur. Phys. J. A 20, 317 (2004) [arXiv:hep-ph/0212347]; K. Pumsa-ard, V. E. Lyubovitskij, T. Gutsche, A. Faessler and S. Cheedket, Phys. Rev. C 68, 015205 (2003) [arXiv:hep-ph/0304033]; T. Inoue, V. E. Lyubovitskij, T. Gutsche and A. Faessler, Phys. Rev. C 69, 035207 (2004) [arXiv:hep-ph/0311275]; K. Khosonthongkee, V. E. Lyubovitskij, T. Gutsche, A. Faessler, K. Pumsa-ard, S. Cheedket and Y. Yan, J. Phys. G 30 (2004) 793 [arXiv:hep-ph/0403119].

[23] J. Gasser and H. Leutwyler, Phys. Rept. 87 (1982) 77; Nucl. Phys. B 250 (1985) 465.

[24] R. Tegen, R. Brockmann and W. Weise, Z. Phys. A 397 (1982) 339. 


\section{FIGURES}

Fig.1: Tree-level diagrams contributing to Compton scattering on a quark.

Figs.2-6: One-body meson-cloud diagrams in Compton scattering.

Figs.7-8: Two-body meson-cloud diagrams in Compton scattering.

Fig.9: Proton electric polarizability $\alpha_{E}^{p}$ : The dotted and the dashed curves are the results of the pion loops without and with inclusion of the excited states. The total value is shown by the solid line. The shaded region shows the experimental results including error bars [5].

Fig.10: Neutron electric polarizability $\alpha_{E}^{n}$ : Otherwise as in Fig.9.

Fig.11: Proton magnetic polarizability $\beta_{M}^{p}$ : The dotted and dashed curves describe the results of the pion loops without and with inclusion of the excited states, respectively. The dotted-dashed line is the contribution from the tree-level diagrams (excited intermediate quark states). The total value is shown by the solid line. The shaded region shows the experimental results including error bars [5].

Fig.12: Neutron magnetic polarizability $\beta_{M}^{n}$ : Otherwise as in Fig.11.

Fig.13: Contribution of the tree-level diagrams (Fig.1) [excited intermediate quark states] to $\beta_{M}^{p}$. The dotted, dashed, dotted-dashed, two-dotted-dashed and two-dashed-dotted lines stand for the results of the individual excited states $1 p_{1 / 2}, 1 p_{3 / 2}, 1 d_{3 / 2}, 1 d_{5 / 2}$ and $2 s_{1 / 2}$.

Fig.14: Contribution of the tree-level diagrams (Fig.1) [excited intermediate quark states] to $\beta_{M}^{n}$. Otherwise as in Fig.13.

Fig.15: Contribution of the meson-cloud diagram (Fig.2) [excited intermediate quark states] to $\alpha_{E}^{p}$. The dotted, dashed, dotted-dashed, two-dotted-dashed and two-dashed-dotted lines stand for the results of the individual excited states $1 p_{1 / 2}, 1 p_{3 / 2}, 1 d_{3 / 2}, 1 d_{5 / 2}$ and $2 s_{1 / 2}$.

Fig.16: Contribution of the meson-cloud diagram (Fig.2) [excited intermediate quark states] to $\alpha_{E}^{n}$. Otherwise as in Fig.15.

Fig.17: Contribution of the meson-cloud diagram (Fig.2) [excited intermediate quark states] to $\beta_{M}^{p}$. The dotted, dashed, dotted-dashed, two-dotted-dashed and two-dashed-dotted lines stand for the results of the individual excited states $1 p_{1 / 2}, 1 p_{3 / 2}, 1 d_{3 / 2}, 1 d_{5 / 2}$ and $2 s_{1 / 2}$.

Fig.18: Contribution of the meson-cloud diagram (Fig.2) [excited intermediate quark states] to $\beta_{M}^{n}$. Otherwise as in Fig. 17. 


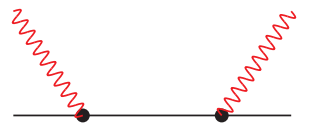

(a)

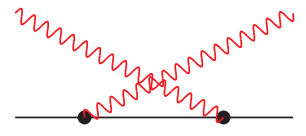

(b)

Fig.1

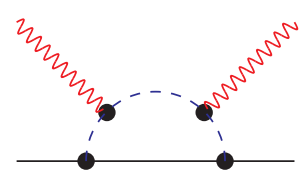

(a)

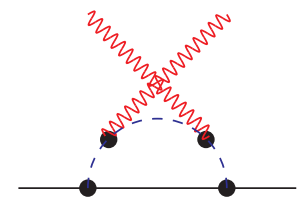

(b)

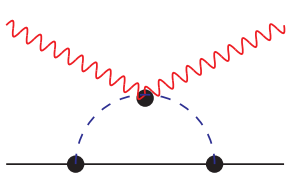

(c)

Fig.2

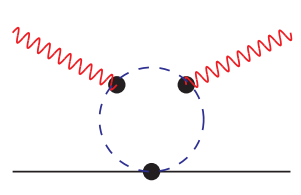

(a)

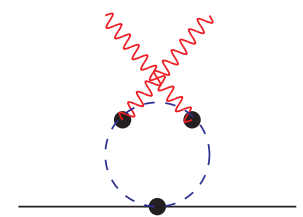

(b)

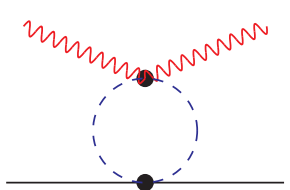

(c)

Fig.3

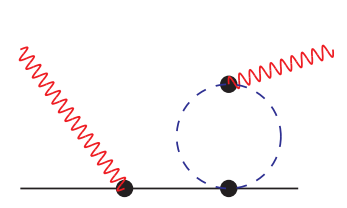

(a)

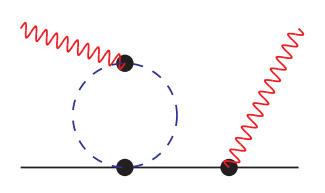

(b)

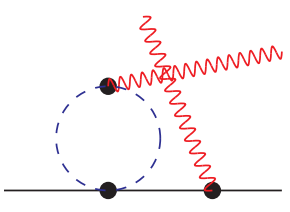

(c)

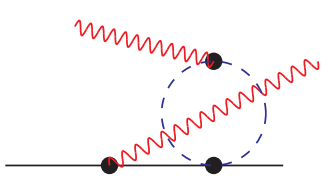

(d)

Fig.4 


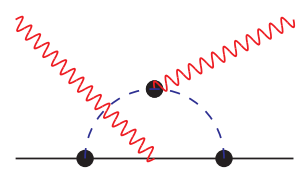

(a)

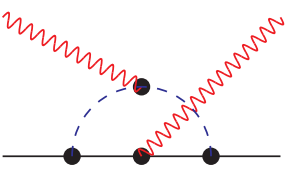

(d)

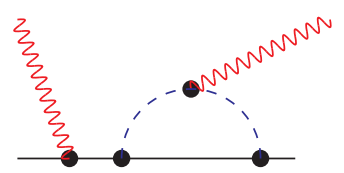

(b)

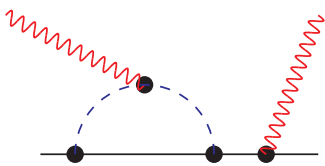

(e)

Fig.5

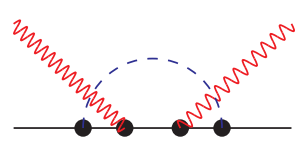

(a)

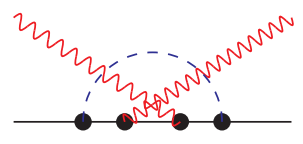

(d)

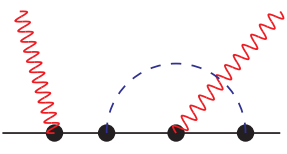

(b)

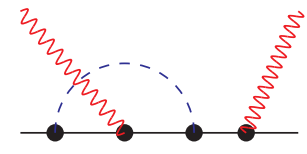

(e)

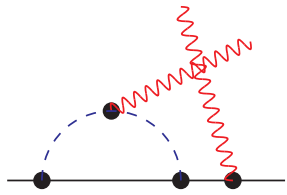

(c)

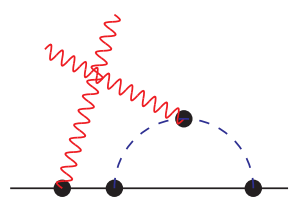

(f)

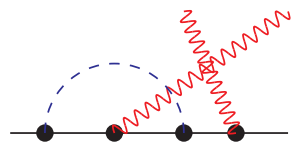

(c)

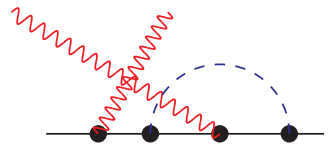

(f)

Fig.6 


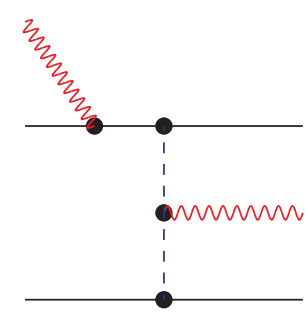

(a)

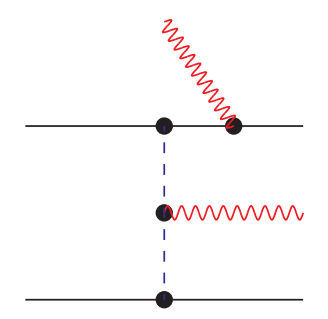

(b)

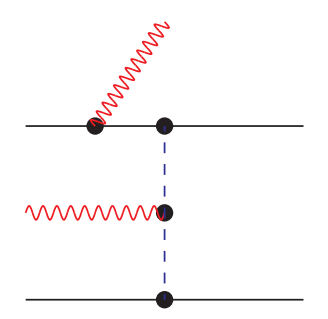

(c)

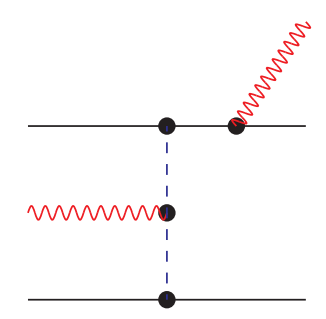

(d)

Fig.7

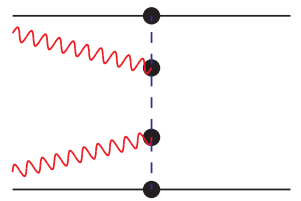

(a)

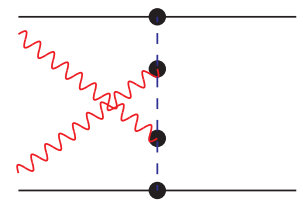

(b)

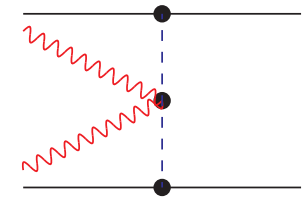

(c)

Fig.8 


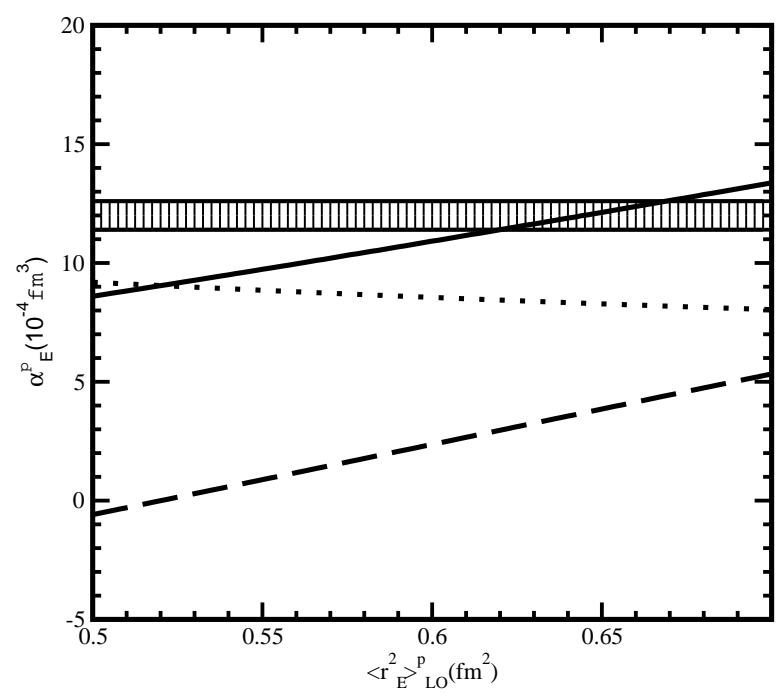

Fig.9

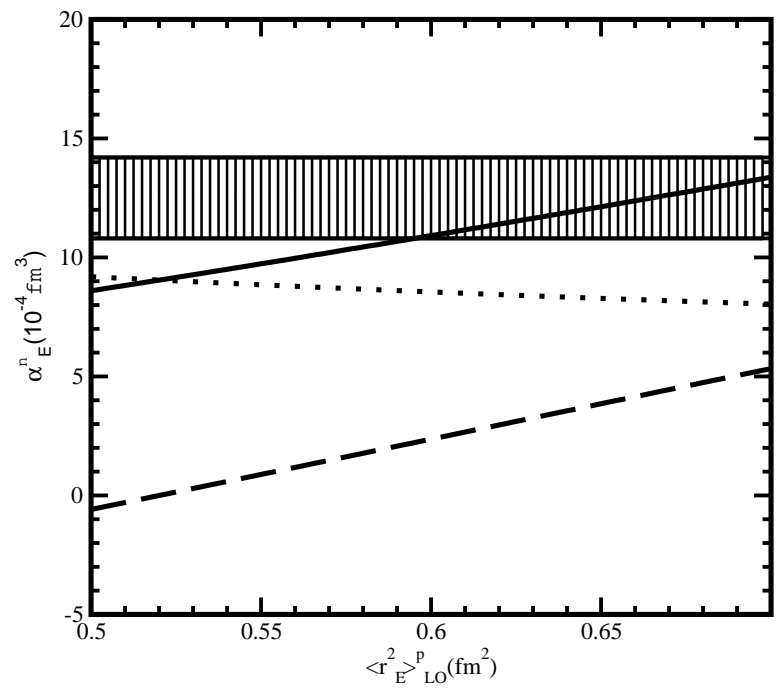

Fig.10 


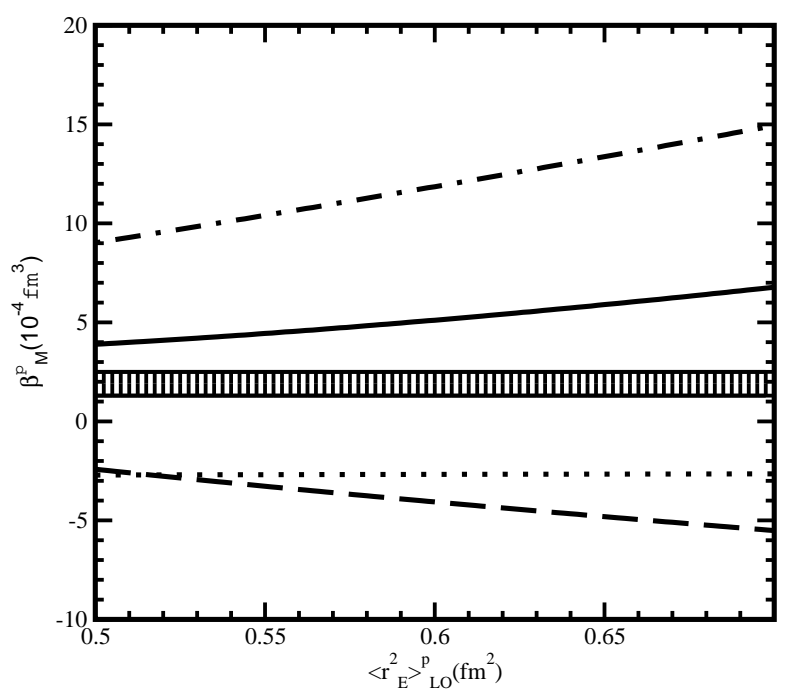

Fig.11

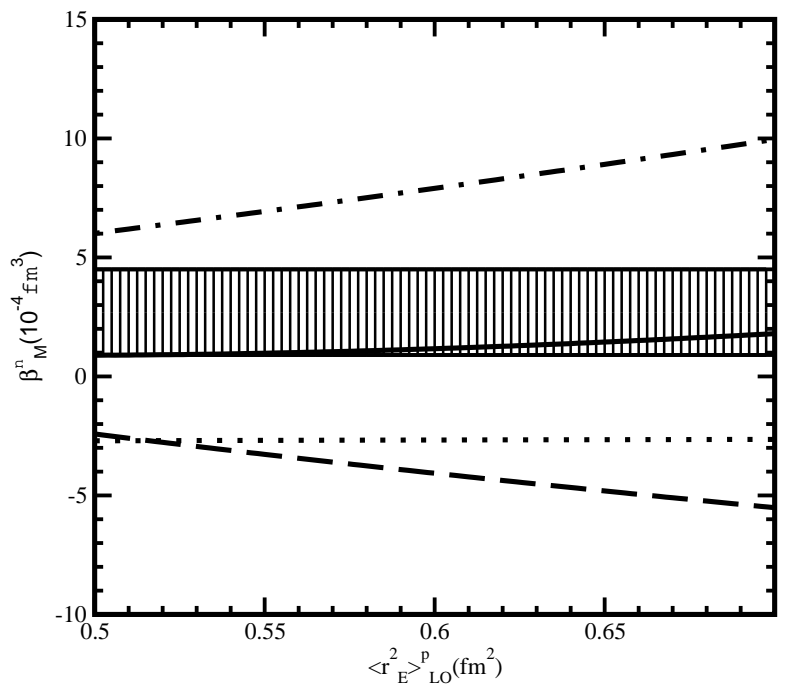

Fig.12 


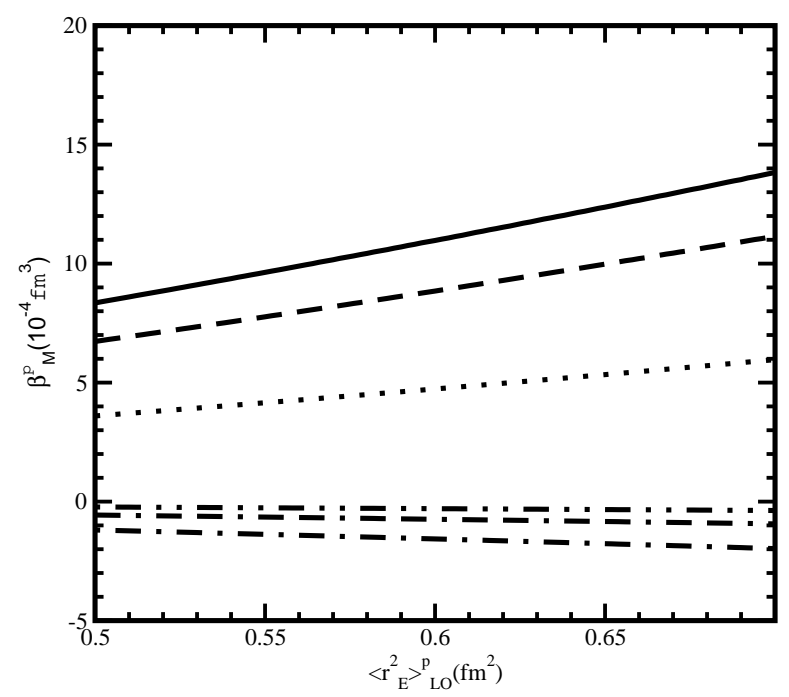

Fig.13

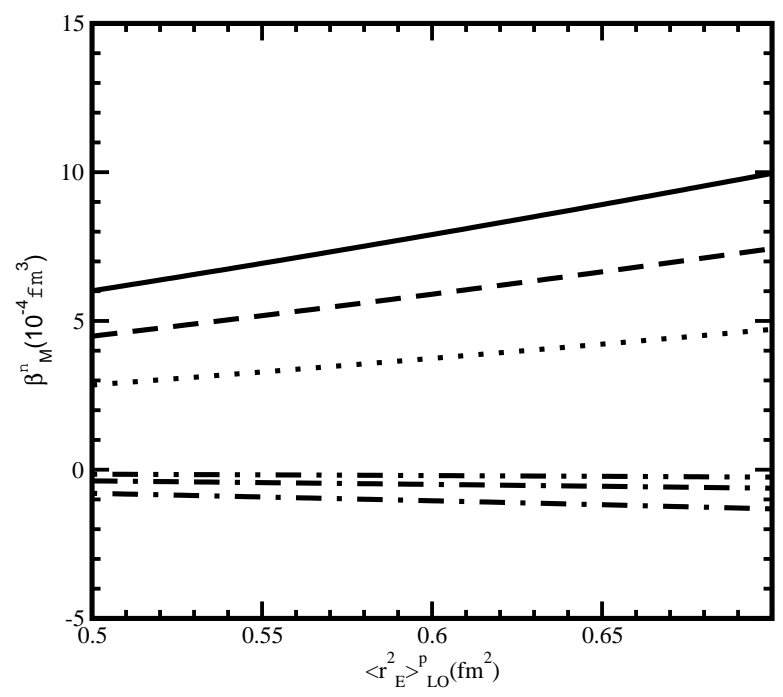

Fig.14 


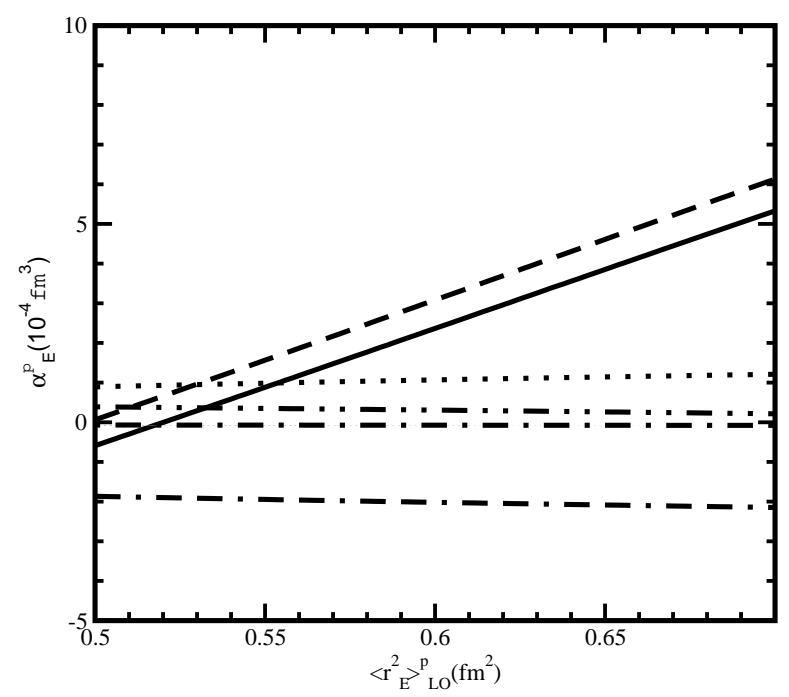

Fig.15

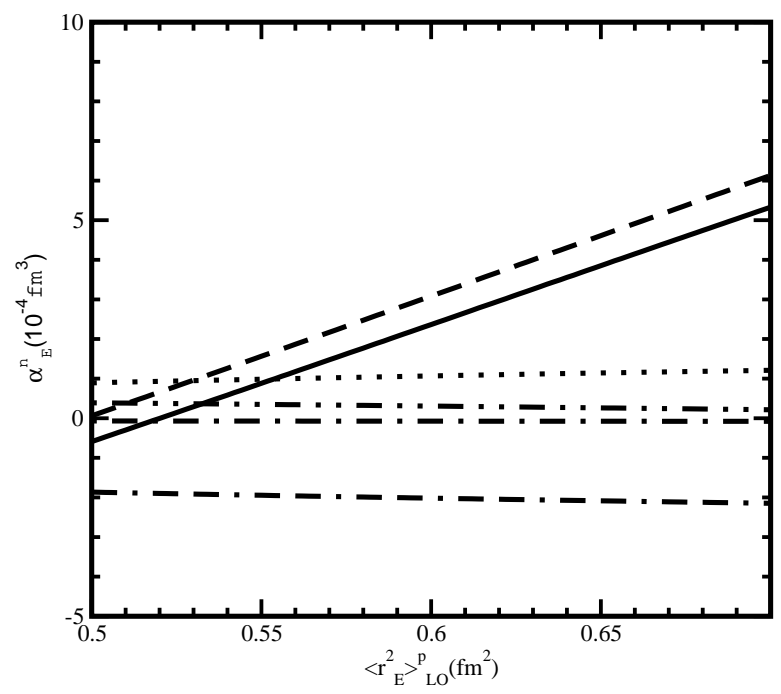

Fig.16 


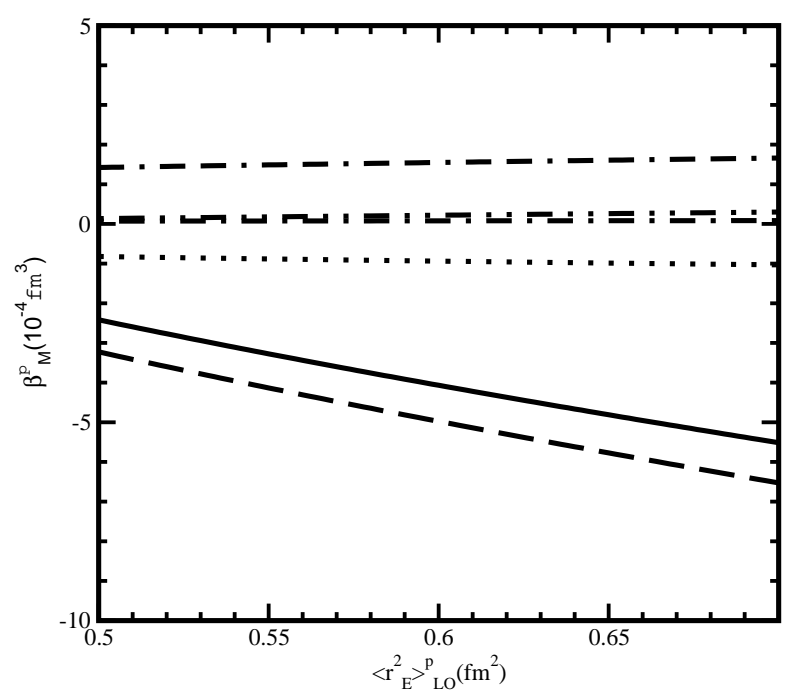

Fig. 17

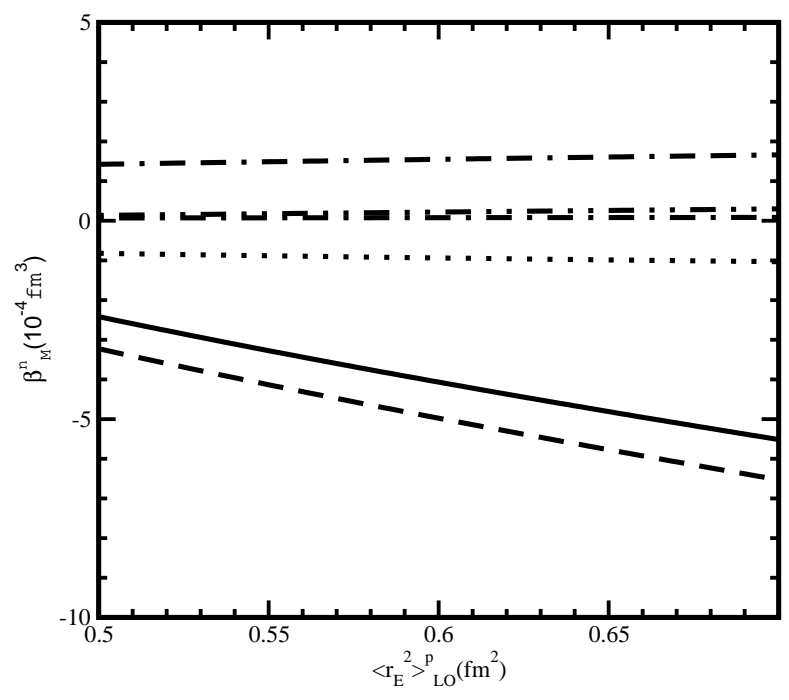

Fig.18 


\section{TABLES}

TABLE I. Results for the nucleon polarizabilities (in units of $10^{-4} \mathrm{fm}^{3}$ ).

\begin{tabular}{||c|cccc||}
\hline \hline Approach & $\alpha_{E}^{p}$ & $\beta_{M}^{p}$ & $\alpha_{E}^{n}$ & $\beta_{M}^{n}$ \\
\hline data [5] & $12.0 \pm 0.6$ & $1.9 \mp 0.6$ & $12.5 \pm 1.7$ & $2.7 \mp 1.8$ \\
\hline \multirow{3}{*}{ ChPT [15] } & 7.9 & -2.3 & 11.0 & -2.0 \\
& $10.5 \pm 2.0$ & $3.5 \pm 3.6$ & $13.6 \pm 1.5$ & $7.8 \pm 3.6$ \\
& 12.6 & 1.26 & 12.6 & 1.26 \\
\hline ChPT + DR [17] & 7.3 & -1.8 & 9.8 & -0.9 \\
\hline PCQM & 10.9 & 5.1 & 10.9 & 1.15 \\
\hline \hline
\end{tabular}

TABLE II. Contributions to $\alpha_{E}^{N}$ and $\beta_{M}^{N}$ from the individual diagrams (in units of $10^{-4} \mathrm{fm}^{3}$ ) evaluated for the central value $<r_{E}^{2}>_{\mathrm{LO}}^{P}=0.6 \mathrm{fm}^{2}$.

\begin{tabular}{||l|cccccccc||}
\hline \hline & $\alpha_{E}^{p}(\mathrm{GS})$ & $\alpha_{E}^{p}(\mathrm{ES})$ & $\alpha_{E}^{n}(\mathrm{GS})$ & $\alpha_{E}^{n}(\mathrm{ES})$ & $\beta_{E}^{p}(\mathrm{GS})$ & $\beta_{M}^{p}(\mathrm{ES})$ & $\beta_{E}^{n}(\mathrm{GS})$ & $\beta_{M}^{n}(\mathrm{ES})$ \\
\hline Fig.1 & 0 & 0 & 0 & 0 & 0 & 11.85 & 0 & 7.90 \\
\hline Fig.2 & 2.03 & 2.37 & 2.03 & 2.37 & -0.65 & -4.07 & -0.65 & -4.07 \\
\hline Fig.3 & 4.26 & 0 & 4.26 & 0 & -1.42 & 0 & -1.42 & 0 \\
\hline Fig.6 & 0 & 0 & 0 & 0 & 0.085 & 0.02 & 0.089 & 0.02 \\
\hline Fig.8 & 2.26 & 0 & 2.26 & 0 & -0.71 & 0 & -0.71 & 0 \\
\hline Total & 8.55 & 2.37 & 8.55 & 2.37 & -2.70 & 7.80 & -2.70 & 3.85 \\
\hline \hline
\end{tabular}

